\title{
EXPOSICIÓN A ESTEREOTIPOS Y CATEGORÍAS DE GÉNERO: CONSECUENCIAS EN TAREAS Y EVALUACIONES
}

\author{
BEATRIZ MONTES BERGES \\ Universidad de Jaén
}

Recibido: 12/02/2009

Aceptado: 03/03/2009

\section{Introducción}

Los estudios sobre los estereotipos y sus mecanismos de activación automática y de aplicación han subrayado que es vital conocer si las personas activas en el fenómeno de la discriminación han sido de alguna manera expuestas a la activación de estereotipos que pudieran ser aplicados posteriormente en una relación interpersonal ${ }^{1}$. En el caso del estudio de la discriminación de género, esta activación de estereotipos se ha realizado experimentalmente, mediante la presentación de las categorías de género, palabras o fotos ${ }^{2}$, o mediante la presentación de adjetivos estereotípicos ${ }^{3}$. De esta manera, parece demostrado que la activación de categorías mediante priming subliminal no sólo tiene efectos

1. BLAIR, Irene V. y BANAJI, Mahrazin R. «Automatic and controlled processes in stereotype priming». Journal of Personality and Social Psychology, 70 (6), (1996), pp. 1142-1163; DiJKSTERHUIS, A. y VAN KNIPPENBERG, A. «The relation between perception and behavior, or how to win a game of trivial pursuit». Journal of Personality and Social Psychology, 74 (4), (1998), pp. 865-877; KAWAKAMI, Kerry y DovidiO, John F. «The reliability of implicit stereotyping». Personality and Social Psychology Bulletin, 27 (2), (2000), pp. 212-225.

2. KAWAKAMI, Kerry y Dovidio, John F. «Confronting prejudice: Effects of prejudice labelling on stereotype activation». Personality of Social Psychology Bulletin, 27 (2002), pp. 212-251; LeMuS, Soledad, MoyA, Miguel, BuKOWSKI, Marcin y LuPiáÑEZ, Juan. «Activación automática de las dimensiones de competencia y sociabilidad en el caso de los estereotipos de género». Psicológica, 29 (2008), pp. 115-132.

3. BlaIR, Irene y BANAJI, Mazharin. Op.cit., experimento 1.

Feminismo/s 13, junio 2009, pp. 105-118 
propios de la mera categorización, sino que también puede provocar dos efectos fundamentales: 1) la activación de los estereotipos y su consiguiente aplicación en evaluaciones e interpretaciones de conductas ${ }^{4}$ y, 2) la activación de representaciones comportamentales relacionados con estas categorías ${ }^{5}$.

Sin embargo, profundizando en el tema y centrándolo en el prejuicio de género, queda por determinar si la activación de categorías de género mediante priming subliminal incrementaría los efectos de la mera categorización, al estar más disponibles estas categorías y los estereotipos relacionados con ellas. De la misma manera, sería de gran interés observar si esta activación podría activar representaciones comportamentales relacionadas con los estereotipos de género, lo cual supondría una aportación totalmente novedosa. En este sentido, tal y como indican Wheeler, Harvis y Petty ${ }^{6}$, encontramos dos posibles vías por medio de las cuales la saliencia (o activación) de estereotipos podría influir en el rendimiento: 1) a través de los mecanismos de amenaza al estereotipo ${ }^{7}$ y 2) por medio de procesos implícitos que relacionan el priming con la conducta ${ }^{8}$. En este trabajo nos decantaremos por estudiar la influencia de la activación automática de estereotipos en la conducta mediante los procesos implícitos, y concretamente en el rendimiento de tareas. Para observar este posible efecto de la activación de estereotipos, se determinaron una tarea verbal y otra matemática, habilidades relacionadas tradicionalmente con los estereotipos de ambos géneros. Aunque todos los estudios apuntan

4. FAzio, Russel H., Jackson, Joni R., Dunton, Bridget C. y Williams, Carol J., FAzio, R. H., JACKSON, J. R., Dunton, B. C. y Williams, C. J. «Variability in automatic activation as an unobtrusive measure of racial attitudes: A bona fide pipeline»? Journal of Personality and Social Psychology, 69 (1995), pp. 1013-1027; HigGins, E. Tory, RHOLES, William S. y JONES, Carl R. «Category accessibility and impression formation». Journal of Experimental Social Psychology, 13 (1977), pp. 141-154; MussweILER, T. «Comparison processes in social judgment: Mechanism and consequences». Psychological Review, 110 (2003), pp. 472-489; Mussweiler, T. y DAMISCH, L. «Going back to Donald: How comparisons shape judgmental priming effects». Journal of Personality and Social Psychology, 95 (6), (2008), pp.1295-1315.

5. Dijksterhuis y Van Knippenberg, Op. Cit; Pause, Bettina M, Ohrt, Anne, Prehn, AleXANDER, y Ferstl, ROMAN. Op. cit.

6. Wheeler, S. Christian, Jarvis, W. Blair G. y Petty, Richard E. «Think unto others: The self-destructive impact of negative racial stereotypes». Journal of Experimental Social Psychology, 37, (2001), pp. 173-180.

7. CAdinu, Mara, MAASS, Anne, Lombardo, Mery y Frigerio, Sara. «Stereotype threat: the moderating role of locus of control beliefs». European Journal of Social Psychology, 36 (2006), pp. 183-197; STEELE, Claude M. «A threat in the air: How stereotypes shape intellectual identity performance». American Psychologist, 52 (1997), pp. 613-629.

8. WheEler, S. Christian y PetTy, Richard E. «The effects of stereotype activation on behavior: A review of possible mechanism». Psychological Bulletin, 127(6), (2000), pp. 797-826. 
que el rendimiento de hombres y mujeres en este tipo de tareas es similar ${ }^{9}$, cuando se encuentran diferencias se asocian con el efecto de la amenaza del estereotipo $^{10}$.

Nuestra hipótesis de partida:

En las tareas de rendimiento verbal y matemática se prevé un efecto principal de la condición priming-no priming, ya que la activación de los estereotipos conllevaría una mejor realización de las tareas que implican habilidades propias de cada género.

\section{Método}

\subsection{Participantes}

Este trabajo se realizó con una muestra de 176 participantes, de los cuales 87 eran hombres y 89 mujeres, y con una media de edad de 21,57 años (SD= $3,37)$. Las mujeres eran alumnas de los primeros cursos de la Facultad de Psicología y de la Escuela Universitaria de Trabajo Social. Los hombres procedían de diversos niveles de estudios, y aquellos que poseían estudios universitarios, pertenecían a distintas carreras.

Como consecuencia de los errores cometidos en la tarea subliminal, que se explicará detenidamente más adelante, y de la existencia de participantes que no habían comprendido correctamente las instrucciones del estudio experimental, el número final de participantes descendió a 148 personas, de las cuales 72 fueron hombres y 76 mujeres. Estos 148 participantes fueron repartidos en las ocho condiciones experimentales.

\subsection{Diseño}

El estudio experimental que aquí se presenta tuvo un diseño $2 \times 2 \times 2$ en el que existían 3 variables independientes fundamentales: la presentación previa de las categorías de género con SOA 15ms (priming vs. no-priming), la situación

9. Hyde, Janet S., Fennema, Elisabeth y LAmon, Susan J. «Gender differences in mathematics performance: A meta-analysis». Psychological Bulletin, 107 (1990), pp. 139-155; HyDE, Janet S. y LinN, Marcia C. «Gender differences in verbal ability: A meta-analysis». Psychological Bulletin, 104, (1988), pp. 53-69; THOMPSOn, Ted y Dinnel, Dale. L. «Poor performance in Mathematics: Is there a basis for a self-worth explanation for women?» Educational Psychology, 27 (3), (2007), pp. 377-399.

10. KELLER, Johannes y DAUENHEIMER, Dirk. «Stereotype threat in the classroom: Dejection mediates the disrupting threat effect on women's math performance». Personality and Social Psychology Bulletin, 29 (2003), pp. 371-381; O’BRIEN, Laurie T. y CRANDALL, Christian S. «Stereotype threat and arousal: Effects on women's math performance». Personality and Social Psychology Bulletin, 29 (2003), pp. 782-789. 
(competición vs. Cooperación) y la tercera, el género, en la que nos centraremos en este artículo.

\subsection{Procedimiento}

Este procedimiento está basado en el que realizan otros estudios ${ }^{11}$ usando las categorías de género como primes y estereotipos de género como palabras estímulo $^{12}$. Esta serie de sustantivos iban precedidos de una palabra que refería bien la categoría «hombre» bien la categoría «mujer» que se presentaba subliminalmente durante $15 \mathrm{~ms}$, tal y como se aconseja en diferentes estudios sobre priming subliminal ${ }^{13}$. Los sustantivos eran adjetivos estereotípicos sustantivados (para impedir que su género pudiera influir en la asociación de éste con la categoría y por tanto, con la respuesta dada): diez adjetivos estereotípicos propios de las mujeres (y de ellos cinco positivos y cinco negativos) y diez adjetivos estereotípicos propios de los hombres (siendo cinco de ellos positivos y cinco de ellos negativos). Concretamente estos sustantivos eran los que aparecen en la tabla 1.

Estos sustantivos fueron seleccionados mediante un estudio piloto realizado con 42 estudiantes de la Escuela Universitaria de Trabajo Social, de los cuales 21 eran hombres y 21 mujeres. En este estudio piloto se les pasó un cuestionario en el que las palabras que se les presentaban eran adjetivos estereotípicos sustantivados que se recogieron a su vez de tres fuentes de la literatura que encontraban una serie de adjetivos estereotípicos de mujeres y hombres: a) Moya y Pérez ${ }^{14}$; b) BSRI ${ }^{15}$; c) ACL ítems de la escala de

11. Croizet, Jean Claude. «Unconscious perception of affective information and its impact on personality trait judgment». Cahiers de Psychologie Cognitive / Current Psychology of Cognition, 17 (1), (1998), pp. 53-70; FAzio, Russell H., SANBonmatsu, David M., Powell, Martha C. y KARDES, Frank R. «On the automatic activation of attitudes». Journal of Personality and Social Psychology, 50 (1986), pp. 229-238; NEELY, James H. «Semantic priming and retrieval from lexical memory: Roles of inhibitiones spreading activation and limited-capacity attention». Journal of Experimental Psychology: General, 106 (1977), pp. 226-254.

12. KAWAKAMI, Kerry y DOVIDIO, John F. Op. cit., «The reliability of implicit stereotyping», experiment 1.

13. BARGH, John A. y CHARTRAND, Tanya L. "Studying the mind in the middle. A practical guide to priming and automaticy research». En H. T. Reis y C. M. Judd (Eds.). Handbook of research methods in social and personality psychology. New York, Cambridge University Press, 2000, pp. 17-39.

14. Moya, Miguel Carlos y PÉREZ, Carlos. «Nuevas perspectivas en el estudio de los estereotipos de género». En P. Valcárcel y J. L. Meliá (Eds.). Métodos y técnicas de intervención. Barcelona, PPU, 1998, pp. 49-60.

15. BEM, Sandra L. «The measurement of psychological androgyny». Journal of Consulting and Clinical Psychology, 42 (1974), pp. 155-162. 
TABLA 1. Sustantivos estereotípicos asignados a mujeres $u$ hombres positivos y negativos utilizados en la tarea de priming subliminal

\begin{tabular}{|c|c|}
\hline & SUSTANTIVOS ESTEREOTÍPICOS \\
\hline \multirow{5}{*}{ POSITIVOS MUJERES } & CALIDEZ \\
\hline & SENSIBILIDAD \\
\hline & LEALTAD \\
\hline & PACIENCIA \\
\hline & IDEALISMO \\
\hline \multirow{5}{*}{ NEGATIVOS MUJERES } & VOLUBILIDAD \\
\hline & INGENUIDAD \\
\hline & IMPREVISIBILIDAD \\
\hline & FRIVOLIDAD \\
\hline & NARCISISMO \\
\hline \multirow{5}{*}{ POSITIVOS HOMBRES } & FRANQUEZA \\
\hline & FUERZA \\
\hline & ASTUCIA \\
\hline & ACTIVIDAD \\
\hline & INVENCIÓN \\
\hline \multirow{5}{*}{ NEGATIVOS HOMBRES } & AGRESIVIDAD \\
\hline & COMPETITIVIDAD \\
\hline & DOMINANCIA \\
\hline & VIOLENCIA \\
\hline & AMBICIÓN \\
\hline
\end{tabular}

masculinidad y feminidad ${ }^{16}$. Y en los que tratamos de evaluar su posible modificación a consecuencia del paso del tiempo. Los resultados indicaron que los sustantivos presentes en la tabla 1 mantenían diferencias significativas de género. El orden de presentación de este cuestionario y de los dos anteriores fue nuevamente contrabalanceado a fin de evitar posibles efectos de orden. De esta manera, en la tarea subliminal se presentaba, en primer lugar, un punto para fijar la atención ${ }^{17}$, con una duración de $90 \mathrm{~ms}$; tras $180 \mathrm{~ms}$ se presentaba la palabra referente a la categoría «hombre» o «mujer» (aleatoriamente

16. HeILBRUN, Alfred B. «Measurement of masculine and feminine sex role identities as independent dimensions». Journal of Consulting and Clinical Psychology, 44 (1976), pp. 183-190.

17. BARgh, John A. y CHARTRAND, Tanya L. Op. Cit; Croizet, Jean Claude. Op. cit. 
seleccionada por el ordenador) durante $15 \mathrm{~ms}$, tras la cual aparecía una máscara compuesta por las consonantes «XQFBZRMQWGBX» ${ }^{18}$ durante $7 \mathrm{~ms}$, para impedir la fijación de las palabras subliminalmente presentadas en la memoria sensorial; después de $495 \mathrm{~ms}$, aparecía en la pantalla un sustantivo estereotípico (también seleccionado aleatoriamente por el ordenador) durante 1995ms tras el cual, el/la participante debería responder: positivo o negativo. La variable dependiente de esta tarea fue el tiempo que el/la participante tardaba en responder, es decir, el tiempo de reacción o la latencia de respuesta (Figura 1). Es importante señalar que también se recogió la respuesta dada a cada sustantivo, para llevar a cabo posteriores análisis que garantizaran el correcto tratamiento de los datos (ver resultados).

El número de ensayos total de la tarea fue de 120, de manera que cada uno de los 20 adjetivos estereotípicos sustantivados aparecía 6 veces, 3 de ellas asociadas a la categoría «hombre» y las otras 3 restantes asociadas a la categoría «mujer». El orden de asociación y de presentación de los estímulos en la pantalla fue completamente aleatorio.

FIGURA 1. Esquema de la tarea de priming subliminal y los tiempos entre cada una de las presentaciones

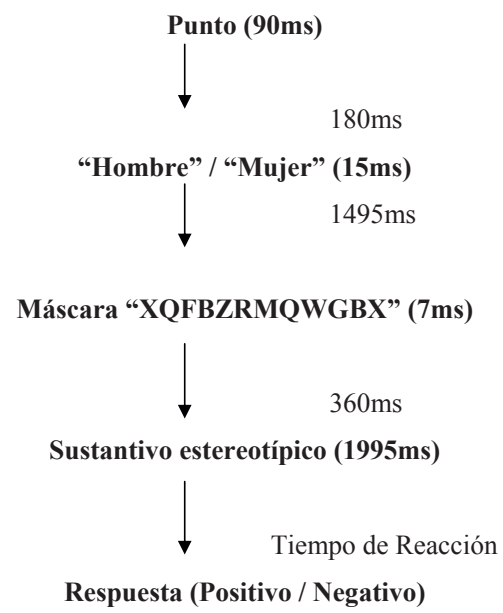

18. BARGH, John A., BAND, Ronald N., LOMBARDi, Wendy J. y TOTA, Mary E. «The additive nature of chronic and temporary sources of construct accessibility». Journal of Personality and Social Psychology, 50 (1986), pp. 869-878. 
A continuación se les daba una breve explicación de las tareas verbal y matemática que tenían que realizar en un tiempo máximo de 7 minutos.

\subsection{Instrumentos.}

En primer lugar, en la tarea de priming subliminal se utilizó un ordenador personal penthium 150, con pantalla de 16 pulgadas. El soporte software fue el programa Medialab con el que se diseñó el procedimiento de priming.

Los instrumentos utilizados en este estudio fueron los siguientes:

1. Tarea verbal: consistía en proporcionar tantos sinónimos como pudieran a todas las acepciones posibles de cada una de las palabras que aparecían a continuación (colaboración, supresión, resolver, realizar, casa, cubículo, estrategia, movilización, incremento, trabajar). La puntuación total de esta prueba se obtenía sumando un punto por cada sinónimo correctamente dado y que estuviera recogido en uno de los siguientes 8 diccionarios. La puntuación mínima sería de 0 y la máxima de tantos puntos como sinónimos correctos pudiesen dar los participantes (en este estudio, el máximo fue 18).

2. Tarea matemática: consistía en resolver 10 operaciones aritméticas, entre las que se encontraban dos sumas, dos restas, dos multiplicaciones, dos divisiones y dos raíces cuadradas que se eligieron para esta prueba. La puntuación total se obtenía sumando un punto por cada operación totalmente correcta, considerándose en el caso de la raíz cuadrada no perfecta, una aproximación de \pm 1 punto. De esta manera, la puntuación mínima sería de 0 y la máxima de 10 . Ambas tareas verbal y matemática debían ser ejecutadas en un tiempo máximo de 7 minutos.

\section{Resultados}

\subsection{Resultados de la Tarea de Priming}

De acuerdo con la teoría relativa al priming y al afecto ${ }^{19}$, el tiempo de reacción para contestar a cada adjetivo estereotípico sustantivado dependería de la congruencia existente entre este adjetivo y la categoría presentada de manera subliminal anteriormente.

El diseño del análisis realizado fue un ANOVA intraparticipantes 2 (categoría presentada en priming subliminal: «hombre»vs. «mujer») x 2 (congruencia vs. incongruencia de los adjetivos estereotípicos con las categorías

19. BARGH, John A., ChEn, Mark y BURROws, Lara. «Automaticy of social behavior: Direct effects of traits construct and stereotype activation on action». Journal of Personality and Social Psychology, 71 (1996), pp. 230-244. 
anteriormente presentadas). Los resultados de tales análisis mostraron que existían diferencias significativas en los tiempos de reacción de los participantes (tabla 2), de manera que cuando las palabras estímulo o targets que se presentaban eran congruentes con los primes, el tiempo de reacción era menor que si no lo eran (tabla 3). Esto ocurría con independencia de que la categoría que aparecía en la pantalla fuera «hombre» o «mujer».

TABLA 2. Diferencia de medias de los tiempos de reacción para las categorías «mujer»y «hombre» con palabras estímulos congruentes o incongruentes a las categorías.

\begin{tabular}{|c|c|c|c|c|}
\hline PRIMES & TARGETS & $\mathbf{t}$ & $\mathrm{df}$ & Sign \\
\hline \multirow{2}{*}{ MUJERES } & poshom-posmuj & 5,74 & 83 & 0,0000 \\
\cline { 2 - 5 } & neghom-negmuj & 3,43 & 83 & 0,0010 \\
\hline \multirow{2}{*}{ HOMBRES } & poshom-posmuj & $-4,54$ & 83 & 0,0000 \\
\cline { 2 - 5 } & neghom-negmuj & $-5,89$ & 83 & 0,0000 \\
\hline
\end{tabular}

TABLA 3. Medias de los tiempos de reacción para las categorías «mujer» y «hombre» con estereotipos femeninos o masculinos.

\begin{tabular}{|c|c|c|c|c|}
\hline \multirow{2}{*}{} & & \multicolumn{3}{|c|}{ PRIMES } \\
\hline \multirow{3}{*}{ TARGETS } & MUJERES & HOMBRES & Total \\
\cline { 2 - 5 } & Femeninos & 953,43 & 1021,03 & 987,23 \\
\cline { 2 - 5 } & Masculinos & 971,31 & 912,27 & 941,79 \\
\cline { 2 - 5 } & Total & 962,37 & 966,65 & 964,51 \\
\hline
\end{tabular}

Los resultados de esta tarea de priming son importantes en cuanto a determinar la influencia de la presentación subliminal de categorías sobre la latencia de respuesta ante palabras congruentes o incongruentes con ellas. El siguiente paso consistía en evaluar si esta influencia ejercería sobre los estereotipos una presión lo suficientemente fuerte, como para que las respuestas ante las siguientes tareas manifestaran diferencias significativas entre los participantes que habían realizado la tarea de priming y por lo tanto manifestaban esta disponibilidad (condición priming) y los participantes que no la habían realizado (condición «no-priming»). 


\subsection{Tareas de rendimiento verbal y numérico}

Para el análisis de estos datos, se realizaron dos ANOVAs 2(condición: priming vs. no-priming) x 2(situación: competición vs. cooperación) x 2(género: hombre vs. mujer), en el que las variables dependientes fue el tiempo de realización de las tareas verbal y matemática, no pudiendo sobrepasar los 7 minutos.

Con respecto a la tarea verbal, los análisis arrojaron que las mujeres puntuaban más alto que los hombres ( $\mathrm{M}=10.06$ vs. $\mathrm{M}=8.2)$, confirmando una diferencia significativa atribuible al género $(F(1,148)=5.87 ; p<.02)$ ). Además, se encontró una interacción entre las variables: género y condición priming- no-priming $(\mathrm{F}(1,148)=22.70 ; \mathrm{p}<.000)$, de manera que en la condición de priming las mujeres puntuaban más en la tarea verbal que los hombres $(\mathrm{F}(1,86)=60.77$; $\mathrm{p}<.000)$, mientras que esta diferencia no existía en la condición de no-priming $(\mathrm{F}(1,58)=1.21 ; \mathrm{p}<.27)$.

En relación con la tarea matemática se observó que los hombres puntuaban más alto $(M=5.5$ vs. $M=4.86)$, confirmando, de nuevo, una diferencia significativa atribuible al género $(\mathrm{F}(1,148)=9.51 ; \mathrm{p}<.002)$. En cuanto a las interacciones entre las variables, se encontró una interacción entre el género y la condición priming-no priming $(F(1,148)=6.42 ; \mathrm{p}<.01)$, de manera que en la condición de no-priming los hombres obtenían mayores puntuaciones en la prueba matemática que las mujeres $(\mathrm{F}(1,59)=.92 ; \mathrm{p}<.004)$, mientras que no se encontraron estas diferencias en la condición de priming $(F(1,87)=.2$; $\mathrm{p}<.66)$.

\section{Discusión}

En este estudio se han utilizado dos tipos de medida: medidas de rendimiento (pruebas verbal y matemática) y medidas de actitudes (cuestionarios de emociones y sentimientos). Estas medidas fueron tomadas, dependiendo de la condición experimental a la que pertenecieran los participantes.

Los análisis muestran diferencias significativas entre los tiempos de reacción de las asociaciones congruentes frente a los tiempos de reacción de asociaciones no congruentes en las asignaciones por género. Así se encontró que cuando los participantes respondían a estereotipos asignados a un género tras la presentación subliminal de la categoría del mismo género, el tiempo de reacción empleado en dar la respuesta era significativamente menor que cuando los estereotipos y la categoría asignada por género no coincidían. De estos resultados se puede deducir que la presentación de las categorías activaba los estereotipos relacionados con ellas, haciéndolos más accesibles, de 
manera que cuando después se presentaban los estereotipos se necesitaba menos tiempo para procesarlos y dar una respuesta según éstos.

Sin embargo, los objetivos de nuestro estudio no se limitaban a confirmar un mero efecto de facilitación de las categorías de género presente en trabajos anteriores ${ }^{20}$ sino que además se proponía observar si estas activaciones influían en el rendimiento de las tareas verbales y matemáticas, de manera que los participantes se comportaran según los estereotipos: los hombres mejores en la prueba matemática y las mujeres mejores en la tarea verbal. Estos datos se deben a la influencia de la condición de priming sobre el rendimiento de las tareas.

Tal y como se propone en la literatura sobre activación de estereotipos, estos resultados pueden explicarse considerando que la activación subliminal de las categorías de género que se presentan activa a su vez a los estereotipos relacionados con estas categorías ${ }^{21}$. Dentro de estos estereotipos se encuentran las diferentes habilidades (verbal y matemática) para las que tradicionalmente uno de los dos sexos se cree mejor que el otro.

Sin embargo la dirección de estas interacciones es diferente entre sí y en relación con las previsiones. Así, en la tarea verbal se encuentra, tal y como se anticipaba, que cuando los participantes tuvieran activadas las categorías de género por la tarea de presentación subliminal, las mujeres puntuarían más y los hombres menos conforme a sus estereotipos. Sin embargo, en la tarea matemática, ocurría lo contrario: cuando los participantes no han realizado la tarea de priming, se encuentra que los hombres puntúan más y las mujeres menos. Estos resultados, en contra de la hipótesis de partida, quizás puedan deberse a que los estereotipos sobre las habilidades especiales de cada género están cambiando, percibiéndose cada vez más a las mujeres como buenas en tareas matemáticas y, o científicas, tradicionalmente consideradas masculinas. Así, podría ser que los estereotipos de género, en constante evolución, tiendan actualmente a reflejar que las mujeres no son malas en tareas científicas (matemáticas) porque de hecho hay ya muchas mujeres que se dedican profesionalmente a estos menesteres, pero que siguen teniendo una habilidad especial de comunicación interpersonal (verbal) que no tienen los hombres. De

20. BAnajI, Mahzarin R. y Hardin, Curtis D. «Automatic stereotyping». Psychological Science, 7, (1996), pp.136-141; KaWAKAMI, Kerry y Dovidio, John, F. Op. cit.

21. HigGins, E. Tory y BREND, C. Miguel. «Accessibility and applicability: Some «activation rules» influencing judgment». Journal of Experimental Social Psychology, 31 (1995), pp. 218-243; LEPORE, Lorella y BROWN, Ruppert. «Category and stereotype activation: Is prejudice inevitable?» Journal of Personality and Social Psychology, 72 (2), (1997), pp. 275-287. 
hecho y en este sentido, es interesante observar que los estudios muestran que cuando se mide el rendimiento verbal o matemático de hombres y mujeres sin que sean expuestos a expectativas o estereotipos previos, no se encuentran diferencias a partir de 1983, coincidiendo con la modificación de la socialización del género. De esta manera, podría explicarse que la activación de las categorías de género supusiera la activación de una mayor habilidad para las mujeres en responder a pruebas verbales, pero no supusiera una mayor habilidad para los hombres en responder a pruebas matemáticas.

Además, también se encuentra una interacción entre la condición priming-no priming y el género sobre las medidas de actitudes.

Así, los resultados muestran que la influencia de la activación de los estereotipos es más fuerte que las expectativas personales de cada individuo sobre las habilidades en las tareas ${ }^{22}$. De esta manera las conclusiones que aquí se presentan ratifican los datos obtenidos por los estudios sobre cognición social. En este sentido, la relevancia de nuestro estudio es clara.

A pesar de ello, somos conscientes de algunas de sus limitaciones. Entre ellas, cabe destacar el nivel de ansiedad que producen las tareas y el diferente grado de habilidad personal de cada uno de los participantes. Ambas limitaciones son susceptibles de ser medidas y por tanto, en cierto modo controlables.

\section{Referencias bibliográficas}

BANAJI, Mahzarin R., Hardin, Curtis D., BANajI, M. R. y HARDin, C. D. «Automatic stereotyping». Psychological Science, 7 (1996), pp. 136-141.

BARGH, John A., BAND, RONALD N. LOMBARdi, Wendy J. y Tota, Mary E. «The additive nature of chronic and temporary sources of construct accessibility». Journal of Personality and Social Psychology, 50 (1986), pp. 869-878.

BARGH, John A., CHEN, Mark y BURROWS, Lara. «Automaticy of social behavior: Direct effects of traits construct and stereotype activation on action». Journal of Personality and Social Psychology, 71 (1996), pp. 230-244.

BARGH, John A. y CHARTRAND, Tanya L. «Studying the mind in the middle. A practical guide to priming and automaticy research». En H. T. Reis y C. M. Judd (Eds.). Handbook of research methods in social and personality psychology. New York, Cambridge University Press, 2000, pp. 17-39.

22. StAngor, Charles, CARr, Christine y KIANG, Lisa. «Activating stereotypes undermines task performance expectations». Journal of Personality and Social Psychology, 75 (5), (1998), pp. 1191-1197. Wout, Daril A., SHiH, Margaret J., JACKSON, James S., SELLERS, Robert M. «Targets as perceivers: How people determine when they will be negatively stereotyped». Journal of Personality and Social Psychology, 96 (2), (2009), pp. 349-362.

Feminismo/s 13, junio 2009, pp. 105-118 
BEM, Sandra L. «The measurement of psychological androgyny». Journal of Consulting and Clinical Psychology, 42 (1974), pp. 155-162.

BLAIR, Irene V. y BANAJI, Mahrazin R. «Automatic and controlled processes in stereotype priming». Journal of Personality and Social Psychology, 70 (6), (1996), pp. 1142-1163.

Cadinu, Mara, MaAss, Anne, Lombardo, Mery y Frigerio, Sara. «Stereotype threat: the moderating role of locus of control beliefs». European Journal of Social Psychology, 36 (2006), pp. 183-197.

CroizeT, Jean Claude. «Unconscious perception of affective information and its impact on personality trait judgment». Cahiers de Psychologie Cognitive / Current Psychology of Cognition, 17 (1), (1998), pp. 53-70.

DiJKSTERHUIS, Ap y VAN KNIPPENBERG, Ad. «The relation between perception and behavior, or how to win a game of trivial pursuit». Journal of Personality and Social Psychology, 74 (4), (1998), pp. 865-877.

FAZIO, Russell. H., JACKSOn, J. R., Dunton, B. C. y Williams, C. J. «Variability in automatic activation as an unobtrusive measure of racial attitudes: A bona fide pipeline?» Journal of Personality and Social Psychology, 69 (1995), pp. 1013-1027.

Fazio, Russell H., Sanbonmatsu, David M., Powell, Martha C. y Kardes, Frank R. «On the automatic activation of attitudes». Journal of Personality and Social Psychology, 50 (1986), pp. 229-238.

Heilbrun, Alfred B. «Measurement of masculine and feminine sex role identities as independent dimensions». Journal of Consulting and Clinical Psychology, 44 (1976), pp. 183-190.

Higgins, E. Tory y BREnd, C. Miguel. «Accessibility and applicability: Some «activation rules» influencing judgment». Journal of Experimental Social Psychology, 31 (1995), pp. 218-243.

Higgins, E. Tory, RHOLES, William S. y JONES, Carl R. «Category accessibility and impression formation». Journal of Experimental Social Psychology, 13 (1997), pp. 141-154.

HydE, Janet S. y LinN, Marcia C. «Gender differences in verbal ability: A metaanalysis». Psychological Bulletin, 104 (1988), pp. 53-69.

Hyde, Janet S., FEnNEMA, Elisabeth y LAMON, Susan J. «Gender differences in mathematics performance: A meta-analysis». Psychological Bulletin, 107 (1990), pp. 139-155.

KAWAKAMI, Kerry y Dovidio, John F. «The reliability of implicit stereotyping». Personality and Social Psychology Bulletin, 27 (2), (2000), pp. 212-225.

- «Confronting prejudice: Effects of prejudice labelling on stereotype activation». Personality of Social Psychology Bulletin, 27 (2002), pp. 212-251. 
KELLER, Johannes y DAUENHEIMER, Dirk. «Stereotype threat in the classroom: Dejection mediates the disrupting threat effect on women's math performance». Personality and Social Psychology Bulletin, 29 (2003), pp. 371-381.

LEMUs, Soledad, MoyA, Miguel, BuKOWSKI, Marcin y LuPIÁÑEZ, Juan. «Activación automática de las dimensiones de competencia y sociabilidad en el caso de los estereotipos de género». Psicológica, 29 (2008), pp. 115-132.

LEPORE, Lorella y BROWN, Ruppert. «Category and stereotype activation: Is prejudice inevitable?». Journal of Personality and Social Psychology, 72 (2), (1997), pp. 275-287.

MoyA, Miguel Carlos y PÉREZ, Carlos. «Nuevas perspectivas en el estudio de los estereotipos de género». En P. Valcárcel y J. L. Meliá (Eds.). Métodos y técnicas de intervención. Barcelona, PPU, 1998, pp. 49-60.

MussweILER, Thomas. "Comparison processes in social judgment: Mechanism and consequences». Psychological Review, 110 (2003), pp. 472-489.

MussweIler, Thomas y DAmisch, Lyssan. «Going back to Donald: How comparisons shape judgmental priming effects». Journal of Personality and Social Psychology, 95 (6), (2008), pp. 1295-1315.

NeEly, James H. «Semantic priming and retrieval from lexical memory: Roles of inhibitionless spreading activation and limited-capacity attention». Journal of Experimental Psychology: General, 106 (1977), pp. 226-254.

O'BRIEN, Laurie T. y CRANDALL, Christian S. «Stereotype threat and arousal: Effects on women's math performance». Personality and Social Psychology Bulletin, 29(2003), pp. 782-789.

Pause, Bettina M., OHrt, Anne, Prehn, Alexander, y Ferstl, Roman. «Positive Emotional Priming of Facial Affect Perception in Females is Diminished by Chemosensory Anxiety Signals». Chemical Senses, 29 (9), (2003), pp. 797-805.

STEELE, Claude M. «A threat in the air: How stereotypes shape intellectual identity performance». American Psychologist, 52 (1997), pp. 613-629.

ThOMPSOn, Ted y DinNel, Dale. L. «Poor performance in Mathematics: Is there a basis for a self-worth explanation for women?». Educational Psychology, 27 (3), (2007), pp. 377-399.

Wheeler, S. Christian, Jarvis, W., Blair G y Petty, Richard E. «Think unto others: The self-destructive impact of negative racial stereotypes». Journal of Experimental Social Psychology, 37 (2001), pp. 173-180.

WheEler, S. Christian y PeTty, Richard E. «The effects of stereotype activation on behavior: A review of possible mechanism». Psychological Bulletin, 127(6), (2000), pp. 797-826. 